\title{
New Methods for Evaluating Students' Answerscripts Using Vague Values
}

\author{
Hui-Yu Wang ${ }^{1}$ and Shyi-Ming Chen ${ }^{2}$ \\ ${ }^{1}$ Department of Education, National Chengchi University, Taipei, Taiwan, R. O. C. \\ ${ }^{2}$ Department of Computer Science and Information Engineering, National Taiwan University of \\ Science and Technology, Taipei, Taiwan, R. O. C.
}

\begin{abstract}
In this paper, we present two new methods for evaluating students' answerscripts using vague values, where the evaluating marks awarded to the questions in the students' answerscripts are represented by vague values. The vague mark awarded to each question of a student's answerscript can be regarded as a vague set, where each element in the universe of discourse belonging to the vague set is represented by a vague value. The proposed methods can evaluate students' answerscripts in a more flexible and more intelligent manner.
\end{abstract}

Keywords: Vague Sets, Vague Grade Sheets, Generalized Vague Grade Sheets, Index of Optimism, Fuzzy Sets.

\section{Introduction}

In recent years, some methods [1], [2], [7], [9]-[12], [14]-[17] for students' evaluation have been presented. In [1] and [7], the fuzzy marks awarded to answers in the students' answerscripts are represented by fuzzy sets [18].However, if we can allow the marks awarded to the questions of the students' answerscripts to be represented by vague values [13], then there is room for more flexibility.

In this paper, we present two new methods for evaluating students' answerscripts using vague values, where the evaluating marks awarded to the questions in the students' answerscripts are represented by vague values. The vague mark awarded to each question of a student's answerscript can be regarded as a vague set, where each element in the universe of discourse belonging to a vague set is represented by a vague value. The proposed methods can evaluate students' answerscripts in a more flexible and more intelligent manner.

\section{Vague Sets and Vague Values}

A vague set $\tilde{A}[13]$ in the universe of discourse $U$ is characterized by a truth-membership function $t_{\tilde{A}}$ and a false-membership function $f_{\tilde{A}}$, where $t_{\tilde{A}}: U \rightarrow[0,1], f_{\tilde{A}}$ : $U \rightarrow[0,1], t_{\hat{A}}\left(u_{i}\right)$ is a lower bound of the grade of membership of $u_{i}$ derived from the evidence for $u_{i}$, $f_{\tilde{A}}\left(u_{i}\right)$ is a lower bound of the negation of $u_{i}$ derived from the evidence against $u_{i}, t_{\tilde{A}}\left(u_{i}\right)+f_{\tilde{A}}\left(u_{i}\right) \leq 1$, and $u_{i} \in U$. The grade of membership of $u_{i}$ in the vague set $\tilde{A}$ is bounded by a subinterval $\left[t_{\tilde{A}}\left(u_{i}\right), 1-f_{\tilde{A}}\left(u_{i}\right)\right]$ of $[0,1]$. The vague value $\left[t_{\tilde{A}}\left(u_{i}\right), 1-f_{\tilde{A}}\left(u_{i}\right)\right]$ indicates that the exact grade of membership $\mu_{\hat{A}}\left(u_{i}\right)$ of $u_{i}$ is bounded by $t_{\tilde{A}}\left(u_{i}\right) \leq \mu_{\tilde{A}}\left(u_{i}\right) \leq 1-f_{\tilde{A}}\left(u_{i}\right)$, where $t_{\tilde{A}}\left(u_{i}\right)+f_{\tilde{A}}\left(u_{i}\right) \leq 1$. If the universe of discourse $U$ is a finite set, then a vague set $\tilde{A}$ of the universe of discourse $U$ can be represented as follows:

$$
\tilde{A}=\sum_{i=1}^{n}\left[t_{\widetilde{A}}\left(u_{i}\right), 1-f_{\widetilde{A}}\left(u_{i}\right)\right] / u_{i} .
$$

In this paper, we present the concept of the expected truth value $E(X)$ of a vague value $X$. Let $X$ be a vague value, where $X=\left[t_{x}, 1-f_{x}\right], t_{x}$ denotes the degree of truth, $f_{x}$ denotes the degree of false, $t_{x} \in[0,1]$, $f_{x} \in[0,1]$, and $t_{x}+f_{x} \leq 1$. Let $f_{x}^{*}=1-f_{x}$, i.e., $\quad$ the vague value $X=\left[t_{x}, 1-f_{x}\right]=\left[t_{x}, f_{x}^{*}\right]$. Then, the unknown part $N(X)$ of the vague value $X$ is defined as follows:

$$
N(X)=f_{x}^{*}-t_{x}=\left(1-f_{x}\right)-t_{x} .
$$

Assume that the index of optimism determined by the evaluator is $\lambda$, where $\lambda \in[0,1]$. If $0 \leq \lambda<0.5$, then the evaluator is a pessimistic evaluator. If $\lambda=0.5$, then the evaluator is a normal evaluator. If $0.5<\lambda \leq 1.0$, then the evaluator is an optimistic evaluator. The larger the value of $\lambda$, the more optimistic the evaluator. The less the value of $\lambda$, the more pessimistic the evaluator. Then, the expected truth value $E(X)$ of the vague value $X$ based on the index of optimism $\lambda$ determined by the evaluator is calculated as follows:

$$
\begin{aligned}
E(X) & =(1-\lambda) \times t_{x}+\lambda \times\left(t_{x}+N(X)\right), \\
& =(1-\lambda) \times t_{x}+\lambda \times\left(t_{x}+f_{x}^{*}-t_{x}\right), \\
& =(1-\lambda) \times t_{x}+\lambda \times\left(t_{x}+\left(1-f_{x}\right)-t_{x}\right), \\
& =(1-\lambda) \times t_{x}+\lambda \times\left(1-f_{x}\right),
\end{aligned}
$$




$$
=(1-\lambda) \times t_{x}+\lambda \times f_{x}^{*},
$$

where $0 \leq t_{x} \leq E(X) \leq f_{x}^{*} \leq 1$ and $f_{x}^{*}=1-f_{x}$.

\section{A New Method for Students' Answerscripts Evaluation Using Vague Values}

In this section, we present a new method for students' answerscripts evaluation using vague values, where the vague marks awarded to the questions of the students' answerscripts are represented by vague values in $[0,1]$. An index of optimism $\lambda$ determined by the evaluator is used to indicate the degree of optimism of the evaluator for evaluating students' answerscripts, where $\lambda \in[0,1]$. If $0 \leq \lambda<0.5$, then the evaluator is a pessimistic evaluator. If $\lambda=0.5$, then the evaluator is a normal evaluator. If $0.5<\lambda \leq 1.0$, then the evaluator is an optimistic evaluator. The larger the value of $\lambda$, the more optimistic the evaluator. The less the value of $\lambda$, the more pessimistic the evaluator. In this paper, eleven satisfaction levels shown in Table 1 are used to evaluate the students' answerscripts regarding a question of a test/examination, where the corresponding vague truth values of these eleven satisfaction levels are also shown in Table 1. Let $T$ be a mapping function for mapping a set of satisfaction levels into their corresponding vague truth values. From Table 1, we can see that

$$
\begin{aligned}
& T(E G)=[1,1], \\
& T(V V G)=[0.90,0.99], \\
& T(V G)=[0.80,0.89], \\
& T(G)=[0.70,0.79], \\
& T(M G)=[0.60,0.69], \\
& T(F)=[0.50,0.59], \\
& T(M B)=[0.40,0.49], \\
& T(B)=[0.25,0.39], \\
& T(V B)=[0.10,0.24], \\
& T(V V B)=[0.01,0.09], \\
& T(E B)=[0,0] .
\end{aligned}
$$

Assume that an evaluator evaluates the students' answerscripts by using a vague grade sheet as shown in Table 2, where $X_{i}$ denotes a vague truth value defined in $[0,1]$ and $1 \leq i \leq 11$. In any row of Table 2 , the columns from the second to the twelfth indicate the vague mark awarded to the answer to the corresponding question shown in the first column, where the vague mark is represented as a vague set. The last (i.e., the thirteenth) column of the vague grade sheet shown in Table 2 indicates the degree of satisfaction evaluated by the proposed method awarded to each question. The box at the bottom of the vague grade sheet shown in Table 2 indicates the total mark awarded to the student.
Table 1. Satisfaction Levels and Their Corresponding Vague Truth Values

\begin{tabular}{l|l}
\hline Satisfaction Levels & Vague Satisfaction Values \\
\hline extremely good $(E G)$ & {$[1,1]$} \\
very very good $(V V G)$ & {$[0.90,99]$} \\
very good $(V G)$ & {$[0.80,0.89]$} \\
good $(G)$ & {$[0.70,0.79]$} \\
more or less good $(M G)$ & {$[0.60,0.69]$} \\
fair $(F)$ & {$[0.50,0.59]$} \\
more or less bad $(M B)$ & {$[0.40,0.49]$} \\
bad $(B)$ & {$[0.25,0.39]$} \\
very bad $(V B)$ & {$[0.10,0.24]$} \\
very very bad $(V V B)$ & {$[0.01,0.09]$} \\
extremely bad $(E B)$ & {$[0,0]$} \\
\hline
\end{tabular}

Table 2. Vague Mark Represented by Vague Values of the Question Q.i in A Vague Grade Sheet

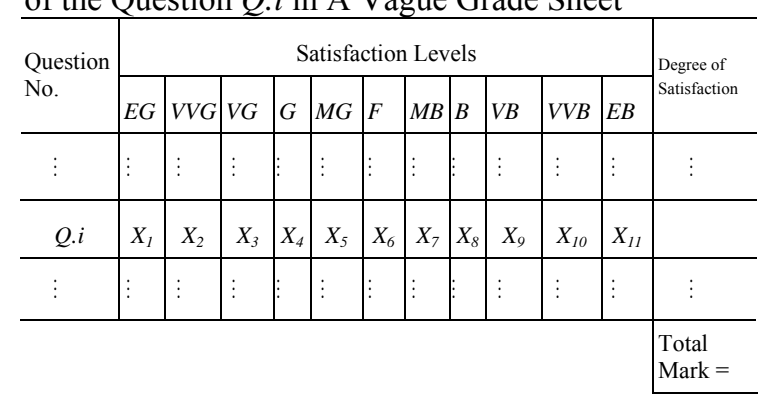

Assume that the vague mark of the question Q.i of a student's answerscript evaluated by an evaluator is as shown in Table 2, where $X_{i}$ is a vague value in the universe of discourse $[0,1]$ and $1 \leq i \leq 11$. Assume that the degree of optimism of the evaluator determined by the evaluator for evaluating students' answerscript is $\lambda$, where $\lambda \in[0,1]$. The proposed method for students' answerscripts evaluation using vague values is now presented as follows:

Step 1: Based on formula (3) and the index of optimism $\lambda$ determined by the evaluator, where $\lambda \in[0$, 1], calculate the expected satisfaction value $E\left(X_{i}\right)$ of each vague satisfaction value $X_{i}$ in the vague grade sheet shown in Table 2, where $E\left(X_{i}\right) \in[0,1]$ and $1 \leq i$ $\leq 11$, as shown in Table 3 .

Table 3. Expected Vague Values of the Vague Mark of the Question Q.i of Table 2

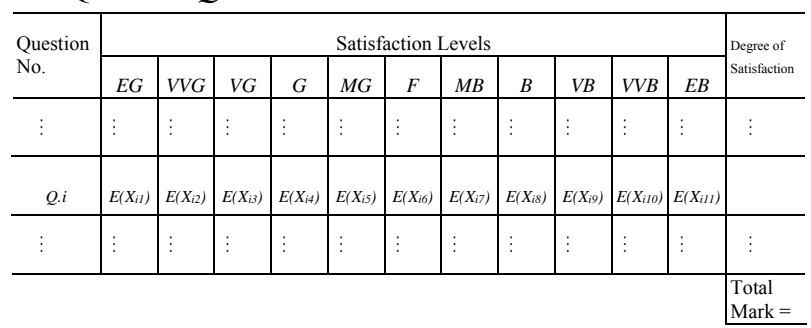


Step 2: From Table 1, we can see that the corresponding vague satisfaction truth values of the satisfaction levels $E G, V V G, V G, G, M G, F, M B, B$, $V B, V V B$ and $E B$ are as follows:: $T(E G)=[1,1]$, $T(V V G)=[0.90,0.99], T(V G)=[0.80,0.89], T(G)=$ $[0.70,0.79], T(M G)=[0.60,0.69], T(F)=[0.50,0.59]$, $T(M B)=[0.40,0.49], T(B)=[0.25,0.39], T(V B)=$ $[0.10,0.24], T(V V B)=[0.01,0.09]$ and $T(E B)=[0,0]$, where $[1,1],[0.90,0.99],[0.80,0.89],[0.70,0.79]$, $[0.60,0.69],[0.50,0.59],[0.40,0.49],[0.25,0.39]$, $[0.10,0.24],[0.01,0.09]$ and $[0,0]$ are vague truth values. Based on formula (3) and the index of optimism $\lambda$ determined by the evaluator, where $\lambda \in[0$, $1]$, calculate the corresponding expected truth value $E(Y)$ of each satisfaction level $Y$ in the vague grade sheet shown in Table 2, where $Y \in\{E G, V V G, V G, G$, $M G, F, M B, B, V B, V V B, E B\}$ and $E(Y) \in[0,1]$. For example, from Table 6 , we can see that $T(V G)=[0.80$, $0.89]$, where $t_{V G}=0.80$ and $1-f_{V G}=0.89$. Assume that the index of optimism $\lambda$ determined by the evaluator is 0.60 (i.e., $\lambda=0.60$ ), then based on formula (3), we can see that the expected truth value $E(V G)$ of the satisfaction level $V G$ is calculated as follows:

$$
\begin{aligned}
E(V G) & =(1-\lambda) \times t_{V G}+\lambda \times\left(1-f_{V G}\right) \\
& =(1-0.60) \times 0.80+0.60 \times 0.89 \\
& =0.32+0.534 \\
& =0.854 .
\end{aligned}
$$

It indicates that the expected truth value of the satisfaction level $V G$ is 0.854 when the index of optimism $\lambda$ determined by the evaluator is 0.60 (i.e., $\lambda$ $=0.60)$. The degree of satisfaction $D(Q . i)$ of the question $Q . i$ of the student's answerscript can be evaluated by the function $D$,

$$
\mathrm{D}(\mathrm{Q} . i)=\frac{E\left(X_{i 1}\right) \times E(\mathrm{EG})+E\left(X_{i 2}\right) \times E(V V G)+\ldots+E\left(X_{i 11}\right) \times E(E B)}{E\left(X_{i 1}\right)+E\left(X_{i 2}\right)+\ldots+E\left(X_{i 11}\right)},
$$

where $E\left(X_{i}\right)$ denotes the expected satisfaction value of the vague satisfaction value $X_{i}, 1 \leq i \leq 11$, and $0 \leq$ $D(Q . i) \leq 1$. The larger the value of $D(Q . i)$, the higher the degree of satisfaction that the question Q.i of the student's answerscript satisfies the evaluator's opinion.

Step 3: Consider the situation that the total mark of a student's answerscript to an examination is 100 marks. Assume that there are $n$ questions to be answered, i.e.,

$$
\text { TOTAL MARKS }=100 \text {, }
$$

Q.1 carries $s_{l}$ marks,

Q.2 carries $s_{2}$ marks,

$$
\text { Q.n carries } s_{n} \text { marks, }
$$

where $\sum_{i=1}^{n} s_{i}=100,0 \leq s_{i} \leq 100$, and $1 \leq i \leq n$. Assume that the evaluated degrees of satisfaction of the questions Q.1, Q.2, $\cdots$, and $Q . n$ are $D(Q .1)$,
$D(Q .2), \cdots$, and $D(Q . n)$, respectively, then the total mark of the student is evaluated as follows:

$$
s_{1} \times D(Q .1)+s_{2} \times D(Q .2)+\cdots+s_{n} \times D(Q . n) .
$$

Put this total mark in the appropriate box at the bottom of the extended fuzzy grade sheet.

\section{A Generalized Method for Students' Answerscripts Evaluation Using Vague Values}

In this section, we present a generalized students' answerscripts evaluation method using vague values. Consider a student's answerscript to an examination of 100 marks. Assume that there are $n$ questions to be answered:

TOTAL MARKS $=100$,

Q.1 carries $s_{l}$ marks,

Q.2 carries $s_{2}$ marks,

Q.n carries $s_{n}$ marks,

where $\sum_{i=1}^{n} s_{i}=100,0 \leq s_{i} \leq 100$, and $1 \leq i \leq n$. Assume that the degree of optimism of the evaluator is $\lambda$, where $\lambda \in[0,1]$. The larger the value of $\lambda$, the more optimistic the evaluator. The less the value of $\lambda$, the more pessimistic the evaluator. Assume that an evaluator evaluates the answers of students' answerscripts using the following four criteria [1]:

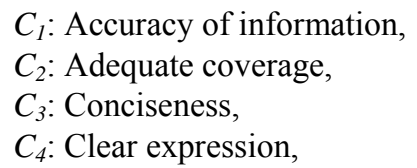

and assume that the weights of the criteria $C_{1}, C_{2}, C_{3}$ and $C_{4}$ are $w_{l}, w_{2}, w_{3}$ and $w_{4}$, respectively, where $0 \leq$ $w_{i} \leq 1$ and $1 \leq i \leq 4$. Furthermore, assume that the evaluator can evaluate each question of students' answerscripts using the above four criteria based on the method described in Section 3. In this case, an evaluator can evaluate students' answerscripts using vague values and the degrees of satisfaction of the question Q.i of a student's answerscript regarding to the criteria $C_{1}, C_{2}, C_{3}$ and $C_{4}$ evaluated by the proposed method presented in Section 3 are $D\left(C_{i l}\right)$, $D\left(C_{i 2}\right), D\left(C_{i 3}\right)$, and $D\left(C_{i 4}\right)$, respectively, where $0 \leq$ $D\left(C_{i l}\right) \leq 1,0 \leq D\left(C_{i 2}\right) \leq 1,0 \leq D\left(C_{i 3}\right) \leq 1,0 \leq D\left(C_{i 4}\right) \leq$ 1 , and $1 \leq i \leq n$. The degree of satisfaction $P(Q . i)$ of the question $Q . i$ of the student's answerscript can be evaluated as follows:

$$
\begin{aligned}
& P(Q . i)= \\
& \quad \frac{w_{1} \times D\left(C_{i 1}\right)+w_{2} \times D\left(C_{i 2}\right)+w_{3} \times D\left(C_{i 3}\right)+w_{4} \times D\left(C_{i 4}\right)}{w_{1}+w_{2}+w_{3}+w_{4}},
\end{aligned}
$$


where $0 \leq P(Q . i) \leq 1$ and $1 \leq i \leq n$. The total mark of the student can be evaluated and is equal to

$$
s_{1} \times P(Q .1)+s_{2} \times P(Q .2)+\cdots+s_{n} \times P(Q . n) .
$$

\section{Conclusions}

In this paper, we have presented two new methods for evaluating students' answerscripts using vague values, where the evaluating marks awarded to the questions in the students' answerscripts are represented by vague values. The vague mark awarded to each question of a student's answerscript can be regarded as a vague set, where each element in the universe of discourse belonging to a vague set is represented by a vague value in $[0,1]$. An index of optimism $\lambda$ determined by the evaluator is used to indicate the degree of optimism of the evaluator. The proposed methods can evaluate students' answerscripts in a more flexible and more intelligent manner.

\section{Acknowledgements}

The authors would like to thank Professor Jason Chihyu Chan, Department of Education, National Chengchi University, Taipei, Taiwan, Republic of China, for providing very helpful comments and suggestions.

\section{References}

[1] R. Biswas, "An application of fuzzy sets in students' evaluation," Fuzzy Sets and Systems, vol. 74, no. 2, pp. 187-194, 1995.

[2] D. F. Chang and C. M. Sun, "Fuzzy assessment of learning performance of junior high school students," Proceedings of the 1993 First National Symposium on Fuzzy Theory and Applications, Hsinchu, Taiwan, Republic of China, pp. 10-15, 1993.

[3] S. M. Chen, "Arithmetic operations between vague sets," Proceedings of the International Joint Conference of CFSA/IFIS/SOFT'95 on Fuzzy Theory and Applications, Taipei, Taiwan, Republic of China, pp. 206-211, 1995.

[4] S. M. Chen, "Measures of similarity between vague sets," Fuzzy Sets and Systems, vol. 74, no. 2, pp. 217-223, 1995.

[5] S. M. Chen, "Similarity measures between vague sets and between elements," IEEE Transactions on Systems, Man, and Cybernetics-Part B: Cybernetics, vol. 27, no. 1, pp. 153-158, 1997.

[6] S. M. Chen, "Evaluating the rate of aggregative risk in software development using fuzzy set theory," Cybernetics and Systems: An International Journal, vol. 30, no. 1, pp. 57-75, 1999.

[7] S. M. Chen and C. H. Lee, "New methods for students' evaluation using fuzzy sets," Fuzzy Sets and Systems, vol. 104, no. 2, pp. 209-218, 1999.

[8] S. M. Chen and J. Y. Wang, "Document retrieval using knowledge-based fuzzy information retrieval techniques," IEEE Transactions on Systems, Man, and Cybernetics, vol. 25, no. 5, pp. 793-803, 1995.

[9] C. H. Cheng and K. L. Yang, "Using fuzzy sets in education grading system," Journal of Chinese Fuzzy Systems Association, vol. 4, no. 2, pp. 81-89, 1998.

[10] T .T. Chiang and C. M. Lin, "Application of fuzzy theory to teaching assessment," Proceedings of the 1994 Second National Conference on Fuzzy Theory and Applications, Taipei, Taiwan, Republic of China, pp. 92-97, 1994.

[11] J. R. Echauz and G. J. Vachtsevanos, "Fuzzy grading system," IEEE Transactions on Education, vol. 38, no. 2, pp. 158-165, 1995.

[12] L. Frair, "Student peer evaluations using the analytic hierarchy process method," Proceedings of 1995 Frontiers in Education Conference, vol. 2, pp. 4c3.1-4c3.5, 1995.

[13] W. L. Gau and D. J. Buehrer, "Vague sets," IEEE Transactions on Systems, Man, and Cybernetics, vol. 23, no. 2, pp. 610-614, 1993.

[14] C. K. Law, "Using fuzzy numbers in education grading system," Fuzzy Sets and Systems, vol. 83, no. 3, pp. 311-323, 1996.

[15] J. Ma and D. Zhou, "Fuzzy set approach to the assessment of student-centered learning," IEEE Transactions on Education, vol. 43, no. 2, pp. 237-241, 2000.

[16] H. Y. Wang and S. M. Chen, "New methods for evaluating the answerscripts of students using fuzzy sets," Proceedings of the 19th International Conference on Industrial, Engineering \& Other Applications of Applied Intelligent Systems, Annecy, France, 2006.

[17] H. Y. Wang and S. M. Chen, "New methods for evaluating students' answerscripts using fuzzy numbers associated with degrees of confidence," Proceedings of the 2006 IEEE International Conference on Fuzzy Systems, Vancouver, BC, Canada, 2006.

[18] L. A. Zadeh, "Fuzzy sets," Information and Control, vol. 8, pp. 338-353, 1965. 